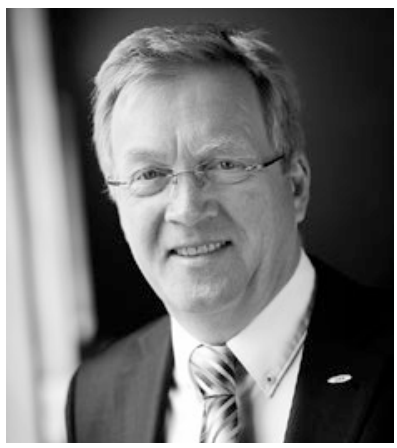

Andreas Westerfellhaus

Präsident des Deutschen Pflegerats
Deutscher Pflegerat e.V.

Bundesarbeitsgemeinschaft

Pflege- und Hebammenwesen

In Kooperation mit

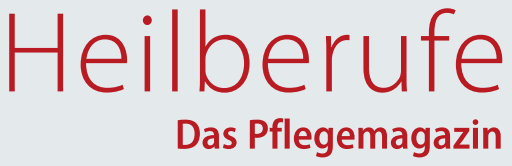

\title{
Editorial
}

\section{Pakt für anständige Löhne}

Der Sozialbereich braucht einen Pakt für anständige Löhne. Das sagt Bundesarbeitsministerin Andrea Nahles. Sie mahnt an, dass die Löhne der Arbeitnehmerinnen und Arbeitnehmer im Bereich Gesundheit und Soziales von der guten Entwicklung abgekoppelt seien. Wer Dienst am Menschen leiste, müsse besser bezahlt werden. Dazu brauchen wir einen „Tarifvertrag Soziales“, so die Ministerin. Der Deutsche Pflegerat unterstützt dies voll und ganz. Deutlich haben die letzten Monate gemacht, dass unserer Berufsgruppe von einigen Politikern wenig Kompetenz zugesprochen wird. $\mathrm{Zu}$ viele und oft fachfremde Akteure haben sich in die Debatte um das Pflegeberufereformgesetz eingemischt. Dabei sind die professionell Pflegenden nicht nur ein „kleines Rädchen“ im System. Wir haben einen Führungsanspruch in der Ausgestaltung des Gesundheitswesens für morgen. Nicht alleine, aber gleichberechtigt mit anderen. 1,2 Millionen Beschäftigte arbeiten in Deutschland im Pflegebereich.

Deren Anliegen sichtbarer zu machen, liegt aber auch in deren eigener Verantwortung. Hierzu brauchen wir die durchgängige Selbstverwaltung und die verpflichtende Mitbestimmung. Dann wird so manche Diskussion anders laufen, als dies heute teilweise der Fall ist. „Still und leise“ war gestern. Mitbestimmen, dabei die Kompetenz der professionell Pflegenden einbringen und das Pflege- und Gesundheitswesen zukunftsorientiert gestalten - das muss unser Ziel sein.

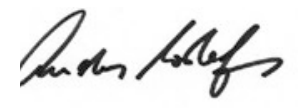

Andreas Westerfellhaus

Präsident des Deutschen Pflegerats

\section{IM FOCUS}

\section{Pflegende müssen mitbestimmen}

Die Berufsgruppe Pflege und Hebammenwesen muss an allen relevanten Gremien des Gesundheitswesens stimmberechtigt beteiligt werden. Das ist eine der Forderungen des Deutschen Pflegerats zur Bundestagswahl in diesem Jahr.

In einer Anhörung des Gesundheitsausschusses des Deutschen Bundestags zur Pflege haben wir dies jetzt erneut untermauert. Konkret geht es um den Qualitätsausschuss nach dem Pflegeversicherungsgesetz. Hier plant der Gesetzgeber, dass alle Entscheidungen der Vertragsparteien nach § 113 SGB XI im Bereich der Qualitätsentwicklung und Qualitätssicherung künftig durch den Qualitätsausschuss getroffen werden, also grundsätzlich auch mit stimmberechtigter Beteiligung der Verbände der Pflegeberufe, mithin des DPR. Diese geplante Klarstellung des Gesetzgebers begrüßen wir ausdrücklich.

Der Deutsche Pflegerat hatte seine Beteiligung an dem Qualitätsausschuss als Vertreter der Verbände der Pflegeberufe gesetzeskonform eingefordert und sich benannt. Eine stimmberechtigte Einbeziehung der Verbände der Pflegeberufe im Qualitätsausschuss war aber bis heute nicht gegeben. Um hier Blockaden zu lösen, muss die Beteiligung der Verbände der Pflegeberufe von einer gesetzlichen Soll- zur Ist-Regelung und damit zwingend werden. Teile der Selbstverwaltung der Einrichtungsträger sollten nicht aus Ängsten vor einem vordergründigen Autonomieverlust einen "verkappten Ausschluss" betreiben, sondern vielmehr im gemeinsamen Interesse die produktive und konstruktive Einbeziehung der Interessen der Pflegeberufe befördern. Das ist unsere Aufgabe als Dachverband der Pflegeberufsorganisationen.

\section{Moritz Ernst}

Leiter der Geschäftsstelle des DPR 